\title{
Relationship between monolayer stacking faults and twins in nanocrystals
}

\author{
A. Hunter ${ }^{1}$, and I. J. Beyerlein \\ Los Alamos National Laboratory \\ Los Alamos, NM 87545, USA
}

\begin{abstract}
A density functional theory-phase field dislocation dynamics model is used to study stress-induced emission of defects from grain boundaries in nanoscale fcc crystals under ambient conditions. The propensity for stable stacking fault formation and the maximum grain size $D_{S F}$ below which a stacking fault is stable are found to scale inversely with the normalized intrinsic stacking fault energy, $\gamma_{I} / \mu b$, where $\mu$ is the shear modulus and $b$ is the value of the Burgers vector. More significantly, we reveal that a grain size smaller than $D_{S F}$ is a necessary but not sufficient condition for twinning. Rather it is shown that deformation twinning additionally scales with $\Delta_{S F E}=\left(\gamma_{U}-\gamma_{I}\right) / \mu b$, where $\gamma_{U}$ is the unstable stacking fault energy. The combined effects of the material $\gamma$-surface and nanograin size for several pure fcc metals are presented in the form of a twinnability map. The findings may provide useful information in controlling nanostructures for improved mechanical performance.
\end{abstract}

Keywords:

partial dislocations, phase field modeling, deformation twin

\section{Introduction}

Deformation twinning has a marked effect on the structural properties of nanocrystalline face-centered cubic (fcc) metals [1]. In such fine, nanometer sized grains, twins, as well as stacking faults and perfect dislocations, are emitted from the grain boundaries [2]. For fcc crystals, perfect dislocations, stacking faults, and twins have some features in common. They are constrained to $\{111\}$ planes, are stable in the fcc crystal, and can accommodate mechanical strain $[3,4,5,6]$. However, these defects are structurally very different. Perfect dislocations are linear defects that do not change the lattice orientation as they glide. Shockley partial dislocations create stacking faults as they glide and these stacking faults are planar defects in which the fcc stacking sequence has been altered. Twins are also planar defects but are comprised of at least two adjacent stacking faults [7]. They reorient the lattice and introduce a twin boundary $[1,5]$. Consequently for the same strain, these three defects do not lead to the same texture, slip activity, and deformation response.

Over the years, extensive experimental, theoretical, and numerical studies have been dedicated to understanding which intrinsic properties, such as the stacking and twin fault energies, and extrinsic properties, such as grain size and grain boundary properties, control which defect is emitted, whether it be perfect dislocations, stacking faults, or twins. Analytical models based on dislocation theory have attempted to dissect step-by-step the development of stacking faults or twins from grain boundaries. Most of them assume that partials are emitted from the same boundary via sequential glide of the same Shockley partial on adjacent planes (referred to as monotonic activation of partials, MAP $[8,9])$. When a single leading partial is emitted and prop- agates across the grain to the opposing grain boundaries without subsequent nucleation of the trailing partial, then a monolayer stacking fault has formed. On the other hand, when the trailing partial nucleates from the same source it corrects the stacking fault and recovers the original stacking. The net displacement corresponds to the glide of a perfect dislocation. However, if instead of the trailing partial, a second leading partial is emitted on the adjacent plane, then a nascent two-layer twin forms.

Validating these defect kinetics has mainly been performed indirectly via post-mortem characterization of deformed nanocrystalline materials and static and dynamic atomic-scale simulations of defect structure or emission. Taken together, observations and atomic-scale simulations clearly indicate that crystal orientation, nanocrystalline grain size, fault energies, or the entire $\gamma$-surface, influence which of these defects is emitted from grain boundaries $[2,10,11,12,13,14,15,16,17]$. In general, when the intrinsic stacking fault energy, $\gamma_{I}$ is low enough, or the nanocrystal small enough, and/or the crystal orientation situated to promote nucleation of the second leading partial nucleation over nucleation of the trailing partial, then twinning becomes likely.

Despite the progress made, there are still many basic questions on twinning in nanocrystals that remain. First, which defect prevails from grain boundaries has yet to be clarified. Further, while it has been shown that both the material $\gamma$-surface and grain size play a role in this selection, their relationship remains elusive [18]. In addition the relative importance of various fault energies, such as the unstable stacking fault energy or twin fault energy, is still not well understood. Last, to date, questions regarding the kinetic pathways taken by defects after they have been emitted from grain boundaries cannot be answered easily via in-situ observation. For this information, 
modeling and simulation have been employed.

Molecular dynamics (MD) simulations and density functional theory (DFT) have been the common numerical tools for relating the $\gamma$-surface to stacking fault formation and twinning. However, both are well known to be limited by very small length and short time scales. In MD, for instance, usually very fine grain sizes $(<<100 \mathrm{~nm})$ and high rates $\left(10^{8} \mathrm{~s}^{-1}\right)$ are simulated. Therefore, while MD simulations have been insightful, it is still desirable to explore stacking fault and twin formation at larger length scales and longer time scales consistent with experiment, where different principles or kinetic pathways could operate. Some meso-scopic simulation tools, such as discrete dislocation dynamics can attain greater length and time scales than MD; however, they tend to make use of rules to model partial dislocations $[19,20]$.

Here we employ a novel meso-scale model, called density functional theory-phase field dislocation dynamics (DFT-PFDD), to explore the collaborative role that the material $\gamma$-surface and grain size play in stress-induced grain boundary emission of stacking faults and twins into nanocrsytals. In the DFT-PFDD calculations, the nucleation and motion of discrete dislocations on crystallographic slip planes are predicted as driven by the minimization of the system energy. Rules are not imposed that could bias which pathways should be followed. To describe the core energy of the defects emitted, this model incorporates the full 3D $\gamma$-surface from DFT into the free energy functional on which the phase field formulation is based. The result is that the most energetically favorable defect and its motion are predicted for a given material and grain size. Whether a leading partial is nucleated and how far it extends are determined by the energetics formulated into the simulation, which involves the strain energy, dislocation core energy, and external work. Likewise, whether a twin forms, is governed by the same governing energetic function. An important benefit of DFT-PFDD is that dislocation motions within large 3D nanocrystals $(\sim 100$ $\mathrm{nm}$ side length) over long periods of time ( $\sim 1$ to several 1000 s) can be simulated.

\subsection{The alternate emission (AE) mechanism for twinning}

In prior work, we used the DFT-PFDD technique [21, 22, 23 ] to study dislocation emission from grain boundaries ledges in $3 \mathrm{D}$ crystals. From these calculations emerged an energetically favorable grain-boundary driven twinning pathway in a 3D grain. Under stress we reveal an alternative mechanism for stacking fault and twin formation; a two-layer twin is formed via partial emissions from opposing grain boundaries. This twinning mechanism accommodates strain yet eliminates backstress. In these respects, this alternate emission (AE) pathway for grain-boundary-driven deformation twinning is distinct from other twinning mechanisms, such as the MAP or random activation of partials (RAP) mechanisms. In MAP, a twin is constructed from sequential glide of identical twinning dislocations emitted onto adjacent planes. MAP could leave ever increasing steps in the grain boundary and consequently, backstresses develop as the twin expands. RAP, the zero-strain twinning mechanism [24], addresses this problem. In RAP, the twinning dislocations emitted from the same boundary have differ- ent Burgers vector that sum to zero. Although no grain boundary backstresses or steps would develop, the RAP mechanism also does not accommodate strain, unlike MAP. The AE mechanism has the potential to be more favorable than MAP or RAP since it both accommodates strain and has an inherent mechanism for removing backstresses.

In this article, we explore the dependencies of the AE twinning mechanism on grain size and intrinsic material properties ( $\gamma$-surface). We show that their effects are in agreement with experiment, yet different from those of other grain-boundary driven mechanisms. In particular, we show that combinations of grain sizes and $\gamma_{I}$ that lead to stable stacking fault formation does not necessarily lead to two-layer twinning. For twinning, the $\Delta_{S F E}=\left(\gamma_{U}-\gamma_{I}\right) / \mu b$ needs to also be sufficiently high.

\section{Model Formulation}

We begin by briefly reviewing some essential elements of the DFT-PFDD model as it applies to fcc metals. Details on the general formulation and derivation of the energy terms can be found in [25, 26, 27, 28, 23].

A DFT-PFDD calculation is based on energy minimization. It predicts, not prescribes, the energetically favorable response that gliding dislocations within a crystal will choose to accommodate the mechanical driving force. Such responses include dislocation nucleation, expansion, and interactions [23, 29, 30, 31, 32].

To model fcc dislocations as partial and full dislocations, the full 3D $\gamma$-surface for a given fcc metal is incorporated into the formulation. In this work the $\gamma$-surfaces for the materials in this study are calculated from DFT using the same PerdewBurke-Ernzerhof (PBE) [33] exchange correlation functional and the projector augmented wave (PAW) potential for the electron-ion interactions [34, 35]. A Monkhorst-Pack scheme [36] was used to construct the Brillouin zone, and the MethfesselPaxton smearing method ( $0.2 \mathrm{eV}$ smearing width) was employed for the integration over the zone [37]. The simulation cells were built using $16\{111\}$ layers, in which the upper eight layers were rigidly shifted in the $\langle 112\rangle$ and $\langle 110\rangle$ directions. Following each shift, the system was relaxed along the $\langle 111\rangle$ direction by minimizing the Hellman-Feyman forces on each atom. For all metals modeled, the energies for each point converged to $10^{-6} \mathrm{eV} /$ atom, and a cut-off energy of $600 \mathrm{eV}$ was adopted. These calculations used a sufficient thickness of vacuum surrounding the shifted slabs, with relaxation in the normal direction of the atomic positions. For more details please refer to [38]. Together, the DFT-PFDD technique permits study of a wide range of fcc metals dislocation core properties based on DFT calculations [21, 22] but at length and time scales much greater than that of DFT or MD alone.

\subsection{Phase Field Dislocation Dynamics (PFDD)}

In general, phase field models track the evolution of one or more scalar order parameters. Specifically in DFT-PFDD, the order parameters $\zeta^{\alpha}(x, t)$ represent the passage of perfect dislocations across each active slip system, $\alpha=12$ for fcc, 
which is defined by the slip plane normal and glide direction $[25,23]$. The plastic distortion results from the motion of dislocations and, hence, can be directly related to the order parameter $[25,39,40]$ :

$$
\beta_{i j}^{p}(x, t)=\sum_{\alpha=1}^{12} b \zeta^{\alpha}(x, t) \delta_{\alpha} s_{i}^{\alpha} m_{j}^{\alpha},
$$

where $b$ is the value of the perfect Burgers vector of the system, $\delta_{\alpha}$ is a Dirac distribution that describes the density of active slip planes, and $s_{i}^{\alpha}$ and $m_{j}^{\alpha}$ are the slip direction and slip plane normal, respectively.

Phase field models evolve systems through minimization of the total system energy $E$. All energy terms contributing to the total $E$ are directly dependent on the order parameters. As in prior works, an additive relationship is assumed between the key energy terms. The total $E$ is then evolved using the Ginzburg-Landau Equation [25, 26, 41, 23]:

$$
\frac{\partial \zeta^{\alpha}(x, t)}{\partial t}=-L \frac{\delta E}{\delta \zeta^{\alpha}(x, t)},
$$

where $L$ is a kinetic coefficient related to dislocation mobility [42].

In the present DFT-PFDD model, $E$ is comprised of three energy terms: the internal elastic energy, external energy, and generalized stacking fault energy (GSFE).

The internal elastic energy, $E^{\text {int }}$ describes both short- and long-range dislocation-dislocation interactions and is the strain energy stored within the system. This term is represented with the classical volume integral

$$
E^{i n t}=\int \frac{1}{2} C_{i j k l} \beta_{i j}^{e}(x) \beta_{k l}^{e}(x) d^{3} x,
$$

where $C_{i j k l}$ is the elastic moduli tensor, and $\beta_{i j}^{e}$ is the elastic distortion. To express Equation 3 in terms of the order parameter via the plastic distortion $\beta_{i j}^{p}$, it is assumed that the displacement gradient can be additively decomposed into the elastic and plastic components. Using stress equilibrium $\left(\sigma_{i j, j}=0\right)$, the displacement field and its gradient can be solved for in terms of $\beta_{i j}^{p}$ [43]. The $\beta_{i j}^{e}$ can then be written as

$$
\beta_{i j}^{e}(x)=-G_{k i, l} *\left(C_{i j m n} \beta_{m n}^{p}(x)\right)_{, j}-\beta_{k l}^{p}(x),
$$

where $G_{i j}$ is the Green's tensor and (*) indicates convolution. By substituting Equation 4 into Equation 3, the internal elastic energy can be expressed in terms of the order parameters. The internal energy energy can be simplified further through application of the complex Fourier transform and convolution theorem. Equation 3 can be expressed as [25, 28, 23].

$$
E^{i n t}(\zeta)=\frac{1}{(2 \pi)^{3}} \int \frac{1}{2} \hat{A}_{\text {mпиv }} \hat{\beta}_{m n}^{p}(k) \hat{\beta}_{u v}^{p *}(k) d^{3} k,
$$

where $\hat{A}_{\text {mпиv }}(k)=C_{\text {mnuv }}-C_{k l u v} C_{i j m n} \hat{G}_{k i}(k) k_{j} k_{l}$, a superposed (^) indicates the Fourier transform, the superscript symbol $\left({ }^{*}\right)$ indicates complex conjugation, and $k_{i}$ is the wave number vector. The degree of anisotropy depends on both the elastic moduli tensor and Green's tensor. For the simulations presented in the following sections, isotropic elasticity has been assumed.

The external energy term, $E^{e x t}$ accounts for the interaction between the dislocations and the applied stress. This term is as follows [25, 28, 23].

$$
E^{e x t}(\zeta)=-\int \sigma_{u v}^{a p p l} \beta_{u v}^{p} d^{3} x,
$$

where $\sigma_{u v}^{a p p l}$ is the externally applied stress. Recall, Equation 1 accounts for the slip direction and slip plane normal. Hence, Equation 6 essentially calculates the resolved shear stress for each active slip system.

The final energy term is the generalized stacking fault energy, $E^{g s f e}$. This term accounts for the energy required to move the dislocation core through the crystal lattice, similar to the Peierls energy [28, 44, 45, 4]. Its functional form greatly depends on the system being studied [46, 25, 41, 28]. To enable the formation, motion and interaction of partial dislocations and extended stacking faults in an fcc metal, this term is formulated to account for the entire fcc material $\gamma$-surface. The $\gamma$-surface is a $3 \mathrm{D}$ material-dependent energy landscape that describes the energy maxima and minima atoms must overcome as they shear pass one another.

As with the other energy terms discussed above, $E^{g s f e}$ must also be directly dependent on the active order parameters. The Burgers vectors of partial dislocations can be expressed as a linear combination of those of perfect dislocations. Since our order parameters correspond to slip by perfect dislocations, $E^{g s f e}$ is generally written as a function of a linear combination of the active order parameters on an active slip plane. For instance, $E^{g s f e}$ for a single glide plane is an explicit function of all three phase variables, $\zeta_{1}, \zeta_{2}$, and $\zeta_{3}$ belonging to this plane.

For simplicity, $E^{g s f e}$ for a single slip plane can be expressed with the following complex Fourier series [47, 48, 28, 23]:

$$
\begin{aligned}
E^{g s f e} & \left(\zeta_{1}, \zeta_{2}, \zeta_{3}\right)=c_{0}+c_{1}\left[\cos 2 \pi\left(\zeta_{1}-\zeta_{2}\right)+\cos 2 \pi\left(\zeta_{2}-\zeta_{3}\right)\right. \\
& \left.+\cos 2 \pi\left(\zeta_{3}-\zeta_{1}\right)\right] \\
& +c_{2}\left[\cos 2 \pi\left(2 \zeta_{1}-\zeta_{2}-\zeta_{3}\right)+\cos 2 \pi\left(2 \zeta_{2}-\zeta_{3}-\zeta_{1}\right)\right. \\
& \left.+\cos 2 \pi\left(2 \zeta_{3}-\zeta_{1}-\zeta_{2}\right)\right] \\
& +c_{3}\left[\cos 4 \pi\left(\zeta_{1}-\zeta_{2}\right)+\cos 4 \pi\left(\zeta_{2}-\zeta_{3}\right)+\cos 4 \pi\left(\zeta_{3}-\zeta_{1}\right)\right] \\
& +c_{4}\left[\cos 4 \pi\left(3 \zeta_{1}-\zeta_{2}-2 \zeta_{3}\right)+\cos 4 \pi\left(3 \zeta_{1}-2 \zeta_{2}-\zeta_{3}\right)\right. \\
& +\cos 4 \pi\left(3 \zeta_{2}-\zeta_{3}-2 \zeta_{1}\right)+\cos 4 \pi\left(3 \zeta_{2}-2 \zeta_{3}-\zeta_{1}\right) \\
& \left.+\cos 4 \pi\left(3 \zeta_{3}-\zeta_{1}-2 \zeta_{2}\right)+\cos 4 \pi\left(3 \zeta_{3}-2 \zeta_{1}-\zeta_{2}\right)\right] \\
& +a_{1}\left[\sin 2 \pi\left(\zeta_{1}-\zeta_{2}\right)+\sin 2 \pi\left(\zeta_{2}-\zeta_{3}\right)+\sin 2 \pi\left(\zeta_{3}-\zeta_{1}\right)\right] \\
& +a_{3}\left[\sin 4 \pi\left(\zeta_{1}-\zeta_{2}\right)+\sin 4 \pi\left(\zeta_{2}-\zeta_{3}\right)+\sin 4 \pi\left(\zeta_{3}-\zeta_{1}\right)\right.
\end{aligned}
$$

The coefficients $c_{0}-c_{4}$ and $a_{1}, a_{3}$ can be determined from either DFT or MD simulations and are discussed in more detail in the next subsection. They are defined as [47]: 


$$
\begin{aligned}
c_{0}= & 0.823\left(4 G-6 G_{1}+6 G_{2}-7.392 G_{3}\right. \\
& \left.+0.804 T+0.804 T_{1}\right) \\
c_{1}= & 0.274\left(-8 G+12 G_{1}-12 G_{2}+14.785 G_{3}\right. \\
& \left.-1.608 T+0.215 T_{1}\right) \\
c_{2}= & 0.091\left(23.072 G-29.138 G_{1}+32.785 G_{2}-42.215 G_{3}\right. \\
& \left.+2.569 T-2.412 T_{1}\right) \\
c_{3}= & 0.137\left(-8 G+12 G_{1}-12 G_{2}+14.785 G_{3}\right. \\
& \left.+0.215 T-1.608 T_{1}\right) \\
c_{4}= & 0.023\left(1.856 G-13.723 G_{1}+6.431 G_{2}-4.277 G_{3}\right. \\
& \left.-0.962 T+3.531 T_{1}\right) \\
a_{1}= & 0.137\left(-32 G+48 G_{1}-48 G_{2}+62.785 G_{3}\right. \\
& \left.-4.608 T-2.785 T_{1}\right) \\
a_{3}= & 0.046\left(17.072 G-19.292 G_{1}+31.923 G_{2}-34.708 G_{3}\right. \\
& \left.+3.341 T-8.354 T_{1}\right)
\end{aligned}
$$

where $T, T_{1}, G, G_{1}, G_{2}, G_{3}$ are certain energetic maxima and minima taken directly from the $\gamma$-surface. To calculate $E^{g s f e}$ over all active slip planes, Equation 7 must be integrated over one slip plane and then summed over all slip planes. The results presented here focus on a single active glide plane ( 3 active slip systems); hence, further discussion of the model formulation will also continue to focus on a single active slip plane.

The 3D PFDD parallel algorithm shows high computational efficiency and scalability for large data sets (domain size on the order of $10^{9}$ ) on up 2,048 cores [39]. For the simulations discussed here the domain size is on the order of $106\left(128^{3}\right.$ grid points) unless otherwise stated with a grid size of one full Burgers vector length. Such a system is large enough to neglect image effects from neighboring dislocations present due to periodic boundary conditions, and still resolve the dislocation lines and their evolution. Periodic boundary conditions are a consequence of the Fourier transforms required to solve for the internal energy (Equation 5 ), and are taken using the Fastest Fourier Transform in the West (FFTW) [49, 50]. Finally the GinzburgLandau Equation is solved using a forward difference numerical derivative with a time step of a half second.

\subsection{Modeling the Material $\gamma$-Surface with DFT-PFDD}

Equation 7 is unique in modeling partial dislocation motion and interactions because it integrates the entire $\gamma$-surface into the governing energetic equation, rather than relying solely on the intrinsic and unstable stacking fault energies. Coefficients $T, T_{1}, G, G_{1}, G_{2}, G_{3}$ in Equation 8 are taken directly from $\gamma$-surface or GSFE curves generated via atomistic methods [47, $48,28,22,23]$. For example, points $G$ and $G_{2}$ represent the intrinsic and unstable stacking fault energies, respectively.

In the following calculations, we employ $a b$ initio DFT to calculate the parameters needed to generate the full material $\gamma$-surface. For consistency, these calculations for all metals examined in this work were carried out using the Vienna Ab-initio Simulation Package (VASP) [51, 52] using the projector augmented wave method within the PBE approximation [33, 22].

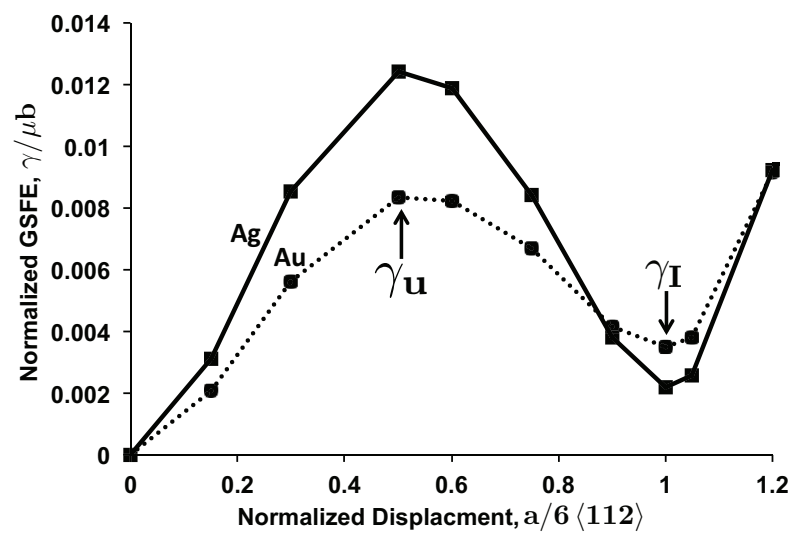

Figure 1: GSFE curves for Au and Ag calculated with ab initio DFT [22]. Arrows highlight the location of the intrinsic and unstable stacking fault energies.

For the elastic moduli of all metals examined here, we also use the results from DFT calculations with the PBE approximation, which are reported by Hunter et al. [22]. As an exception, since the same method produces elastic moduli of Au that noticeably differ from reported experimental values [22], we use measured moduli for $\mathrm{Au}$ in the present calculations.

Figure 1 shows the GSFE curves for Ag and Au. These two metals have nearly the same lattice parameter $a$ and hence Burgers vector value $b$. We see that these curves differ primarily in their normalized unstable stacking fault energy $\gamma_{U} / \mu b$, and only slightly differ in the normalized intrinsic stacking fault energy values. Another interesting feature in these curves is that the normalized intrinsic stacking fault energy for $\mathrm{Au}$ is greater than that for $\mathrm{Ag}$, however this trend reverses for the normalized unstable stacking fault energy. Hence, there is also a noticeable difference in $\Delta_{S F E}=\left(\gamma_{U}-\gamma_{I}\right) / \mu b$.

\subsection{Initial Conditions}

We consider a cuboidal fcc grain with side length $D$, as in Figure 2, bounded by six equivalent grain boundaries. Also, in the next sections, we consider several fcc metals. Table 1 presents the material parameters for all materials discussed. For these calculations, the grain boundaries are assumed impenetrable. Under a uniform applied load, the grain boundaries generate a spatially varying stress field in the grain [23]. This field can nucleate dislocations without an outstanding grain boundary flaw; however, very large stresses would be required to do so. Furthermore, for the nanoscale range of $D$ probed here (up to $100 \mathrm{~nm}$ ), twins have been found to nucleate from pre-existing defects in grain boundaries [53, 54, 55, 56]. To best represent this scenario and isolate the nucleation event, we chose not to insert a priori a particular twinning, partial, or extended full dislocations. Instead, we place ledges within the grain boundaries and allow for the DFT-PFDD energy minimization to predict the dislocation type nucleated and subsequent reaction path for a given grain material and size, $D$. These ledges represent full dislocations that are located on the grain boundary. Numerically, this translates into a small region near the grain boundary 
in which the phase field variable in the appropriate direction (i.e., the x-direction in Figure 2) is initially set to one.

We chose a crystal orientation-applied load relationship such that for an fcc metal many different dislocation types could potentially nucleate: one of six perfect dislocations or six Shockley partial dislocations, where only three of the latter set can potentially build a twin [3]. A uniform shear stress is applied to drive a partial to nucleate from the ledge (Figure 2). To remove any stress-grain size inter-relationships, the applied stress is increased until a partial or partials are emitted and then held constant for the remainder of the simulation. This procedure defines a threshold stress for Shockley partial nucleation, $\sigma_{t h}$. The $\sigma_{t h}$ depends on the ledge size $L$ and not grain size $D$ except at very small grain sizes (10 $\mathrm{nm}$ and less) [57, 23] and hence for the same metal, the same applied stress can be used for all values of $D$. Among different metals, $\sigma_{t h}$ is proportional to the normalized unstable stacking fault energy $\gamma_{U} / \mu b[23,58]$.

To explore the relation between monolayer stacking faults and twins, we consider the three different initial configurations shown in Figure 2. Configuration $\mathrm{A}$ is the most common physical picture modeled, containing a grain boundary ledge on a single slip plane. Similar but with a little more complexity, Configuration B has an additional grain boundary defect on the opposing grain boundary. In this configuration, these defects lie on the same slip plane. Configuration $\mathrm{C}$ is slightly modified from B. It has two opposing grain boundary defects, as in Configuration B; however, the defects lie on adjacent planes. All configurations are compared for the same crystallographic orientation, which is given in Figure 2. As shown, the orientation of the cube with respect to the applied stress state $\sigma_{x z}$ is $x=[10 \overline{1}], y=[111]$, and $z=[\overline{1} 2 \overline{1}]$. Periodic boundary conditions are employed in all three orthogonal directions. Grain boundaries are present on all faces of the crystal cube with width $w=1.25 \mathrm{~nm}$, and modeled as an array of pinned obstacles. The grain boundary regions and hence the phase field variables (initially set to zero) associated with these regions are not allowed to evolve via Equation 2. This will create localized stress fields surrounding the grain boundary regions. Although these initial conditions have been set up to be geometrically symmetric, slight differences in the stress fluctuations near the ledge can cause one partial to emit slightly before the other.

\section{Results}

\subsection{Monolayer fault formation}

We first consider the formation of extended, monolayer stacking faults using Configuration A shown in Figure 2. The following sequence of events is seen for $\mathrm{Ag}$ and $\mathrm{Au}$ for the same grain size $D=37.6 \mathrm{~nm}$. Under an applied stress, the leading partial dislocation nucleates from the ledge. As the leading partial bows out away from the grain boundary, the dislocation line becomes asymmetrical in order to maximize the screw component, which has a lower self-energy in comparison to the edge component $[3,4]$. We observe that the leading partial traverses the entire grain leaving behind an extended monolayer stacking fault. Thus for this grain size $D$ and in both $\mathrm{Ag}$ and $\mathrm{Au}$, a stable

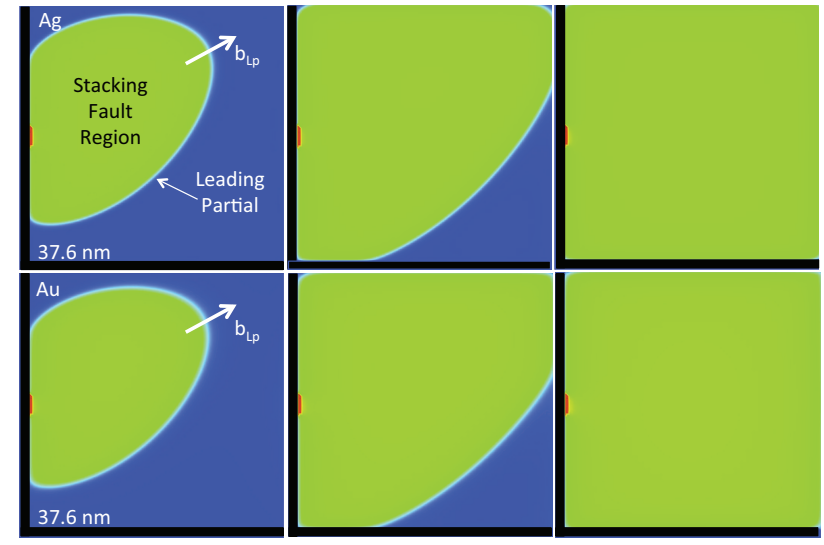

Figure 3: (Color online) Monolayer fault formation in $\mathrm{Ag}$ and $\mathrm{Au}$ for $D=37.6$ $\mathrm{nm}$ grain size. Both metals easily form monolayer (full-grain) stacking faults. Additional PFDD simulations have shown that $\mathrm{Ag}$ and $\mathrm{Au}$ form monolayer stacking faults in grain sizes upwards of $75 \mathrm{~nm}$. Black border regions indicate grain boundaries and green regions indicate stacking fault regions.

monolayer fault can form. Denoting $D_{S F}$ as the maximum grain size below which a full-grain stacking fault is stable, we see that $D_{S F}$ is greater than $37.6 \mathrm{~nm}$ for both $\mathrm{Ag}$ and Au. More simulations for larger grain sizes find that stable full-grain stacking faults can form in these two metals for grain sizes at least as large as $75 \mathrm{~nm}$. Apparently $D_{S F}$ for $\mathrm{Ag}$ and Au are greater than $100 \mathrm{~nm}$. For all grain sizes simulated, the threshold stresses $\sigma_{t h}$ for $\mathrm{Ag}$ and $\mathrm{Au}$ were 0.92 and $0.83 \mathrm{GPa}$, respectively. The difference correlates well with the difference in their $\gamma_{U} / \mu b$ (i.e., Figure 1).

\subsection{Alternate emission mechanism for monolayer fault forma- tion}

We next considered equal-sized grain boundary ledges placed on opposing sides of the grain but on the same glide plane (Configuration B, Figure 2). For Ag and $\mathrm{Au}$ grains of the same size $D=37.6 \mathrm{~nm}$, we observed nucleation of two oppositely signed Shockley partial loops, as shown in Figure 4. Similar to the previously discussed case with only a single grain boundary ledge (Configuration A), the loops assume an asymmetric state in order to maximize the screw component. The leading partial dislocations meet and annihilate each other but leave the entire plane faulted. Therefore, on the chance that steps on opposing grain boundaries coincide on the same plane, an applied stress can drive them to nucleate oppositely signed Shockley partials that join and form a stacking fault (green region in Figure 4). As a consequence of permitting nucleation on both sides of the grain, we observe an interesting AE mechanism for monolayer stacking fault formation. Testing other grain sizes, we find that AE stacking fault formation is possible for $D<D_{S F}$, where $D_{S F}$ is again at least $100 \mathrm{~nm}$ for both $\mathrm{Ag}$ and $\mathrm{Au}$.

\subsection{Alternate emission mechanism for twin formation}

A nascent deformation twin can be created via glide of Shockley partials on adjacent planes. To give the opportunity for twin formation, we repeated these simulations but placed grain 

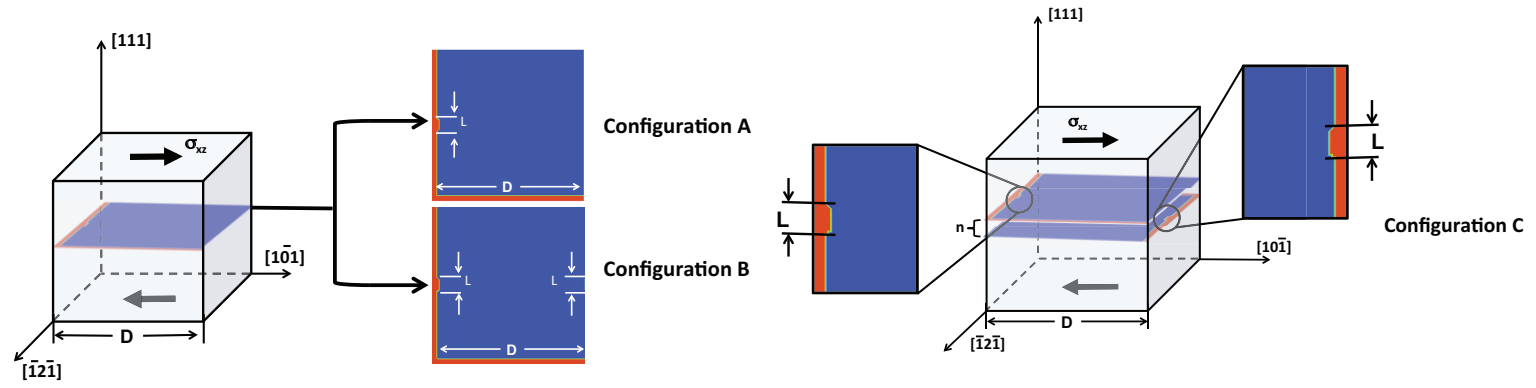

Figure 2: (Color online) Schematic of the 3D simulation set-up showing grain orientation and the externally applied stress state. Configuration A represents a single grain boundary defect. Configuration B shows two grain boundary ledges on the same slip plane but opposing grain boundaries. Finally, Configuration C shows two grain boundary defects on opposing grain boundaries and adjacent slip planes. All ledges have an effective Burgers vector equal to that of a full edge dislocation [10ī].

Table 1: Elastic constants and GSFE parameters (Equation 8) calculated by DFT using the Perdew-Burke-Ernzerhof (PBE) exchange correlation functional [21, 22] The lattice parameter, $a$ has units of $\AA$, the bulk, shear, and young's modulus, $B, \mu$, and $E$, respectively, are in units of GPa, and the GSFE parameters, $A$, $G_{1}, G_{2}$, $G_{3}, G, T_{1}$, and $T_{2}$ have units of $\frac{m J}{m^{2}}$.

\begin{tabular}{|c|c|c|c|c|c|c|c|c|c|c|c|c|}
\hline Material & $a$ & $B$ & $\mu$ & $E$ & $v$ & $A$ & $G_{1}$ & $G_{2}$ & $G_{3}$ & $G$ & $T_{1}$ & $T$ \\
\hline Aluminum & 4.04 & 78.1 & 28.4 & 76.0 & 0.338 & 0 & 68.3 & 151.4 & 175.9 & 140.2 & 190.8 & 334.8 \\
\hline Copper & 3.64 & 137.9 & 54.5 & 144.4 & 0.325 & 0 & 87.5 & 163.7 & 112.8 & 38.5 & 288.5 & 509.4 \\
\hline Gold & 4.17 & 137.1 & 18.8 & 54.0 & 0.434 & 0 & 35.4 & 66.5 & 53.2 & 27.9 & 134.3 & 228.5 \\
\hline Iridium & 3.88 & 347.7 & 222.3 & 549.7 & 0.236 & 0 & 330.1 & 614.9 & 526.8 & 324.4 & 829.6 & 1207.9 \\
\hline Nickel & 3.52 & 195.2 & 99.7 & 255.7 & 0.282 & 0 & 151.2 & 289.0 & 242.6 & 144.6 & 457.3 & 785.8 \\
\hline Palladium & 3.95 & 169.0 & 45.7 & 125.8 & 0.376 & 0 & 97.8 & 197.9 & 186.1 & 138.1 & 283.9 & 459.5 \\
\hline Rhodium & 3.84 & 255.6 & 154.1 & 384.9 & 0.249 & 0 & 242.3 & 454.1 & 355.6 & 184.6 & 648.5 & 962.7 \\
\hline Silver & 4.16 & 89.4 & 27.5 & 74.8 & 0.361 & 0 & 55.1 & 100.4 & 68.2 & 17.8 & 173.6 & 298.2 \\
\hline
\end{tabular}



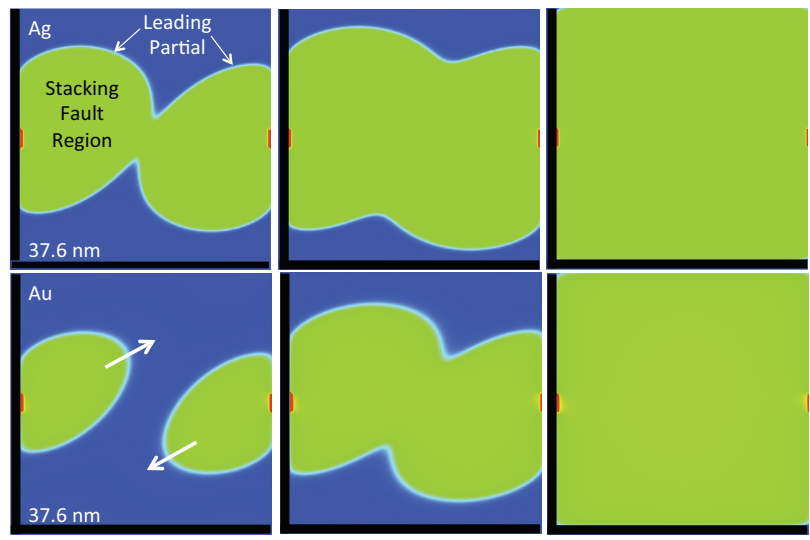

Figure 4: (Color online) Monolayer fault formation through alternate emission in $\mathrm{Ag}$ and $\mathrm{Au} D=37.6 \mathrm{~nm}$ grains. Both metals easily form monolayer (fullgrain) stacking faults. Black border regions indicate grain boundaries and green regions indicate stacking fault regions.

boundary ledges on opposite ends on adjacent planes (Configuration C, Figure 2). Figure 5 shows our results for the $D=37.6 \mathrm{~nm} \mathrm{Ag}$ and Au grains. For these two metals under the same threshold stresses as before, we observe that Shockley partials of opposite signs are emitted from each ledge and driven towards one another. Also as seen previously in simulations with starting Configurations $\mathrm{A}$ and $\mathrm{B}$, we find that once the partial loops get close enough to interact in the center of the grain, they expand outward maximizing the screw component of the loop. After this point, $\mathrm{Ag}$ and $\mathrm{Au}$ deviate in their response.

In $\mathrm{Ag}$, each Shockley partial traverses the grain and forms a stable fault. The final structure is a two-layer twin. This kinetic pathway for twin formation in nanoscale grains differs from the customary mode of layer-by-layer glide of identical twinning dislocations (TDs). Hereinafter we refer to it as the AE mechanism for twin formation. Based on this result, it would appear that when $D<D_{S F}$, then $D$ is sufficient to also form a twin.

In Au, however, the two-layer twin does not form. Upon meeting, they first stop due to their attractive interaction. They may either expand outward or one may push the other, as they aim to accommodate the applied strain. When this evolution eventually becomes energetically unfavorable, the trailing partial is emitted from either one or both ledges, as shown in Figure 5. As a result, perfect dislocations form and their glide accommodates the applied drive. Unlike Ag, for $\mathrm{Au}$, a grain size sufficient to form a full-grain stacking fault is not sufficient to form a twin. Further by changing the grain boundary ledge configuration for the same $D$, we see a stacking fault in one case and full dislocation slip in another.

To explore the possibility that grain size plays a different role in monolayer vs. twin formation, we performed a simulation on an Au crystal much smaller in size $(9.4 \mathrm{~nm})$ also shown in Figure 5. Again, the two-layer twin does not form. As shown, ultimately the trailing partial on the upper glide plane is emitted from the grain boundary ledge and hence, full dislocation slip is favored once again over twin formation. Apparently

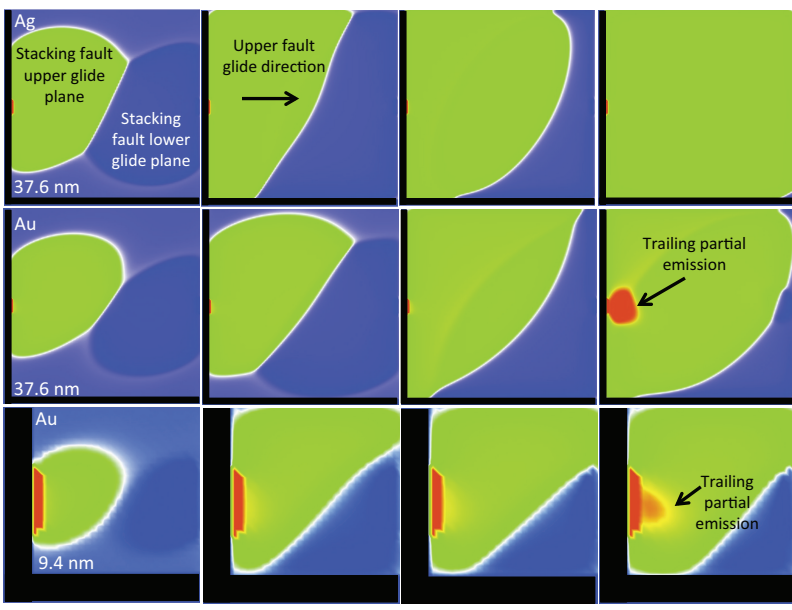

Figure 5: (Color online) Two-layer twin formation through alternate emission for an $\operatorname{Ag} D=37.6 \mathrm{~nm}$ grain. Interestingly for the same grain size in $\mathrm{Au}$, a twolayer twin does not form and a trailing partial dislocation is emitted instead. For a smaller grain size $(D=9.4 \mathrm{~nm})$, Au still does not form a two-layer twin via alternate emission. Rather the upper fault forces the lower fault to shrink, which is then followed by trail emission. Black border regions indicate grain boundaries and green regions indicate stacking fault regions.
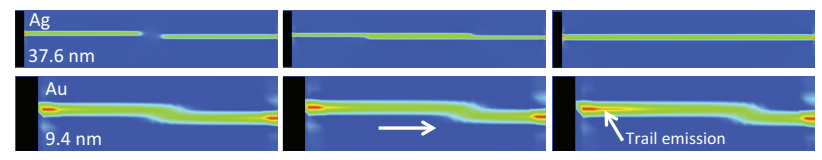

Figure 6: (Color online) Cross-sectional view of two-layer twin formation through alternate emission for an $\mathrm{Ag} D=37.6 \mathrm{~nm}$ grain. A cross-sectional view of expanding stacking faults is also shown for an $\mathrm{Au} D=9.4 \mathrm{~nm}$ grain size. Rather than two-layer twin formation, the Au grain emits a trailing partial dislocation despite the small grain size. Black border regions indicate grain boundaries and green regions indicate stacking fault regions.

shrinking the grain size further below $D_{S F}$ does not promote twinning. Figure 6 shows cross-sectional views of the twolayer stacking fault propagation in the $37.6 \mathrm{~nm} \mathrm{Ag}$ grain, and in the $9.4 \mathrm{~nm}$ Au grain. Here the 'pushing back' of one partial dislocation by the other is clearly depicted.

In short, despite the fact that both metals can form a stable monolayer fault via emissions of partials from grain boundaries, and have a relatively low intrinsic stacking fault energy, one metal forms a twin and the other does not. Therefore, for the AE mechanism, monolayer fault formation does not imply twin formation.

\section{Discussion}

\subsection{Influence of $\gamma$-surface and grain size on monolayer fault formation}

We have shown that monolayer stacking fault formation is a necessary but insufficient condition for twin formation. Further, our results thus far hint that the fault energy landscape (the $\gamma$-surface) affects whether monolayer stacking fault correlates to twin formation. To elucidate this relationship, we carry out simulations to determine $D_{S F}$ and the twinnability of grains 


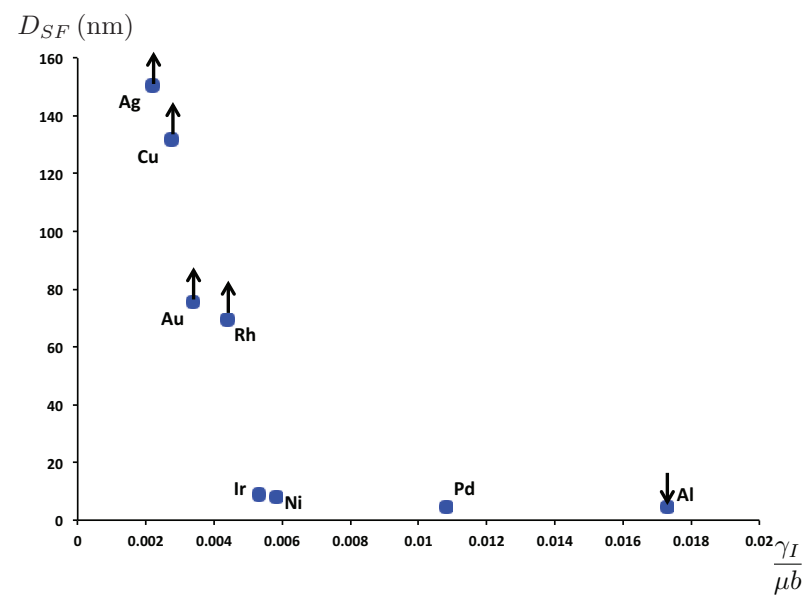

Figure 7: (Color online) The relationship between $D_{S F}$ and $\gamma_{I} / \mu b$ for several fcc metals. Upward arrows indicate that the actual value of $D_{S F}$ lies above the displayed point. Similarly, downward arrows indicate that $D_{S F}$ lies below the displayed points. Clearly the relationship between $D_{S F}$ and $\gamma_{I} / \mu b$ is non-linear.

$D<D_{S F}$ for several fcc metals, varying widely in their fault energy landscapes $[22,23]$. To be systematic in this pursuit, the grain boundary ledge sizes are fixed in all simulations. Second, for the same grain size $D$, the simulation is repeated when the ledges are on the same plane (Configuration $\mathrm{B}$ ) to test for $\mathrm{AE}$ monolayer fault formation or adjacent planes (Configuration $\mathrm{C}$ ) to test for twin formation. Last, the threshold stress is applied corresponding to the minimum stress needed to drive emission of leading Shockley partials from the ledges.

When $D<D_{S F}$, leading partials emitted from grain boundaries form a stable fault across the grain cross section. Above $D_{S F}$ the correcting trailing partial is emitted from the ledge before the leading partial traverses the entire grain. In this case, a stacking fault is not stable. Our DFT-PFDD calculations find that for $\mathrm{Al}$ and $\mathrm{Pd}, D_{S F}$ is $4.5 \mathrm{~nm}(\mathrm{Pd})$ or less (Al) [23]. We do not consider these metals further for twinning. For Ni and Ir, $D_{S F}$ are $7.97 \mathrm{~nm}$ and $8.78 \mathrm{~nm}$, respectively. For $\mathrm{Au}$ and $\mathrm{Rh}$, $D_{S F}$ is greater than $70 \mathrm{~nm}$. Finally, $D_{S F}$ is greater than 100 $\mathrm{nm}$ for $\mathrm{Cu}$ and $\mathrm{Ag}$ [23]. Significantly, we find that $D_{S F}$ scales inversely with $\gamma_{I} / \mu b$ but not with $\gamma_{I}$. A similar inverse scaling with $\gamma_{I} / \mu b$ arises with their equilibrium core widths $w_{0}$ [22]. The maximum grain size $D_{S F}$ for stable monolayer faults from grain boundary emission, however, is generally much greater than the $w_{0}$ of an extended full dislocation in the interior of the grain. Also for $w_{0}$ the scaling with $\gamma_{I} / \mu b$ is linear, whereas for $D_{S F}$, the scaling with $\gamma_{I}$ is not, as shown in Figure 7.

\subsection{Influence of $\gamma$-surface on twin formation}

Calculations to test for twinning with Configuration $\mathrm{C}$ find that twins form in $\mathrm{Ag}, \mathrm{Cu}$, and $\mathrm{Rh}$, provided that $D<D_{S F}$. Twin formation in a $24.6 \mathrm{~nm} \mathrm{Rh}$ grain is shown in Figure 8. In contrast, twins did not form in the remaining metals in spite of $D<D_{S F}$. In fact, these non-twinning metals could not be made to twin by shrinking the grain size $D$ further below $D_{S F}$. Unlike stacking fault formation, the likelihood of twinning does

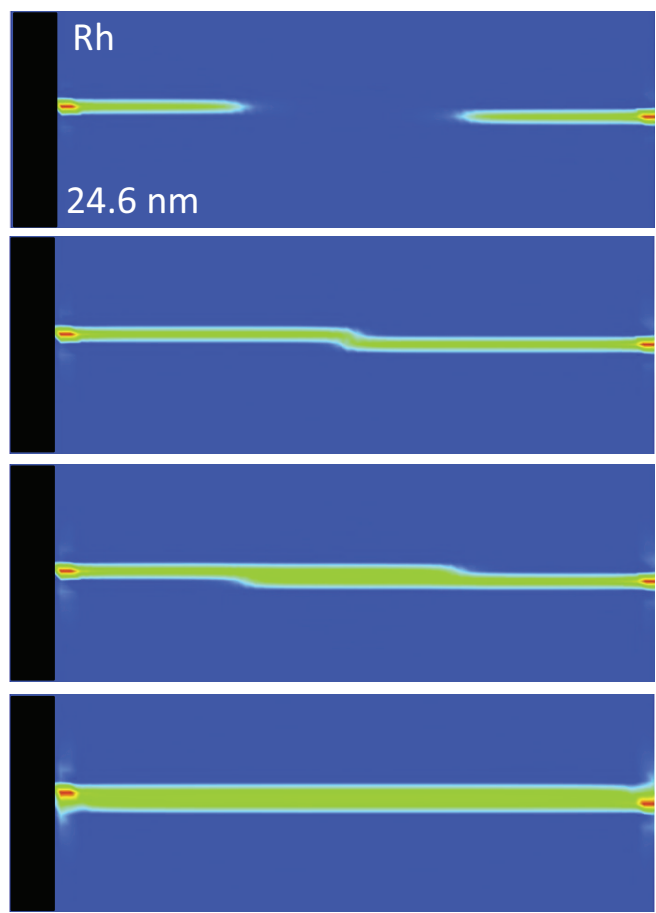

Figure 8: (Color online) Cross-sectional view of two-layer twin formation via the AE mechanism in a $D=24.6 \mathrm{~nm}$ Rh grain. Black border regions indicate grain boundaries and green regions indicate stacking faults.

not depend solely on $\gamma_{I} / \mu b$ or having a sufficiently small grain size. For instance, Au and Ag have nearly the same $\gamma_{I} / \mu b$ and can easily propagate a monolayer fault across the same grain sizes, but one twins easily ( $\mathrm{Ag})$ and the other does not $(\mathrm{Au})$.

We note that these results are consistent with observations reported in the literature. Experimental studies report that deformation twins form easily in nanocrystalline $\mathrm{Ag}$ and $\mathrm{Cu}$ at room temperature. At room temperature, $\mathrm{Ag}$ twins have been reported in grains ranging from a $100 \mathrm{~nm}$ to several microns $[59,60,61]$. Room temperature experiments on $\mathrm{Cu}$ nanostructures have reported twins in grain diameters as large as $150 \mathrm{~nm}$ $[62,59,63]$. Twinning is observed to be relatively difficult in nanocrystalline $\mathrm{Au}$. Twins have been reported in nano-thick $\mathrm{Au}$ films, but these twins were associated with outstandingly large asperities in the boundary [64]. The remaining metals are known to be hard to twin in ambient temperatures and low rates. Crack-tip twinning in Ir has been reported high-rate MD simulations [65]. Experiments only report twins in nano-sized grains of $\mathrm{Al}$ after severe (surface) grinding [14] and in nano-sized $\mathrm{Ni}$ grains at liquid nitrogen temperatures $[66,18]$. Unfortunately, there are no deformation studies on nanocrystalline $\mathrm{Rh}$. In light of our forecasted propensity for deformation twinning in $\mathrm{Rh}$, this phenomenon would be interesting to test.

\subsection{Stacking fault energy dependencies}

For deformation twinning via the AE mechanism, the dependence on the $\gamma$-surface can be understood via a simple model describing the competition between the energy required for stack- 


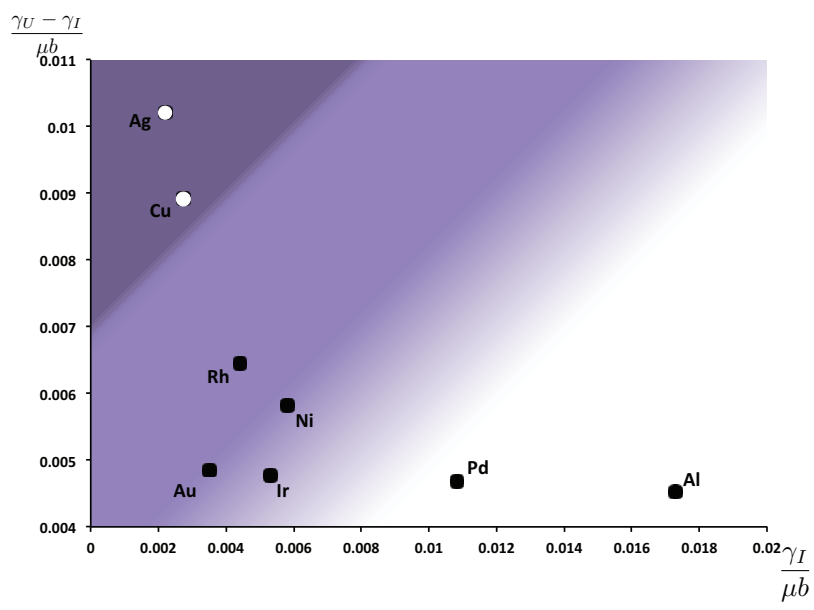

Figure 9: (Color online) Map highlighting four key regions of extended stacking fault formation and two-layer twin formation via the AE mechanism for a wide range of fcc metals. The metals in the upper left-hand corner ( $\mathrm{Ag}$ and $\mathrm{Cu}$ ) are those in which two-layer twin formation is more prevalent. There is a clear dependence on key stacking fault energies from the material $\gamma$-surface, namely the normalized intrinsic stacking fault energy and the difference between the intrinsic and unstable stacking fault energies (also normalized).

ing fault formation and that needed for trailing partial nucleation. Each participating partial must be able to propagate a stacking fault across the grain. At the same time, the activation barrier for trail nucleation must be larger than the attractive interaction energy between two approaching, oppositely signed partials. This physical picture translates to two conditions. First, the grain size and normalized intrinsic stacking fault energy $\gamma_{I} / \mu b$, where $\mu$ is the shear modulus and $b$ the value of the Burgers vector, should be sufficiently small to accommodate a stacking fault area that covers the entire grain crosssection. Second, the energy difference for nucleation of a trail over creating the stacking fault, $\Delta_{S F E}=\left(\gamma_{U}-\gamma_{I}\right) / \mu b$, must also be sufficiently high. Accordingly with both fulfilled, emitted leading partials can propagate a stable stacking fault across the entire grain cross-section without exceeding the energy penalty to provoke emission of a trailing partial.

As a way of elucidating their collaborative effects, Figure 9 shows a map of these two measures plotted against one another for a wide range of pure fcc metals. Achieving both criteria translates to a twinnable region for $\mathrm{AE}$ that lies in the upper left-hand corner. $\mathrm{Ag}$ and $\mathrm{Cu}$ not only lie in this region, but are set apart from the others. Thus, with all else being the same, it is anticipated that the other metals are less likely to produce twins via $\mathrm{AE}$ compared to $\mathrm{Ag}$ and $\mathrm{Cu}$. Rh lies closest to $\mathrm{Cu}$ and $\mathrm{Ag}$ and as shown in Figure 8, can also twin, provided that $D>D_{S F}$. The remaining metals lie further away and are less likely to twin. As a further test, similar ambient simulations were carried out on $\mathrm{Ir}$ and $\mathrm{Al}$ in which only the first criterion is met but not the second. As forecasted, they did not form a two-layer twin. To sum, the trends revealed by the map suggest that twinnability scales with $\Delta_{S F E}$ given that the grain size is sufficiently small $D<D_{S F}$.

\subsection{Comparisons with other twin propensity measures}

Questions of grain size and stacking fault energy dependencies associated with other twinning mechanisms, mostly with the MAP mechanism, remain unsettled. Many twinnability parameters have been devised to indicate the propensity of twinning. They vary in form, function, and the fault energies they consider, some additionally including the unstable and stable twin fault energies [67, 68, 10, 11, 14, 12, 16, 17, 69, 15].

In Kibey et al. [10, 11], a critical twinning stress is defined. According to their model, $\mathrm{Al}, \mathrm{Pd}$, and $\mathrm{Pt}$ would not twin; and $\mathrm{Ag}, \mathrm{Cu}, \mathrm{Au}$, and $\mathrm{Ni}$ would twin. Ir and $\mathrm{Rh}$ were not considered. Other models have defined a twinnability parameter (TP), based on $\gamma_{I}, \gamma_{U}$, and sometimes $\gamma_{U T}$, in which twinning is associated with values of the TP above a certain critical number [68, 15]. Such criteria can, however, be too sensitive to the values chosen for the relevant fault energies $\gamma_{I}, \gamma_{U}$, and $\gamma_{U T}$, which is problematic since there is too much variation in these parameters among different MD and DFT calculations. To make this point, we summarize in Table 2 the values for these TPs using the values of $\gamma_{I}, \gamma_{U}$, and $\gamma_{U T}$ from DFT $[69,22]$. For instance, for the TP from [68], values that lie above unity signify twinning. As shown in Table 2, for all the fcc metals considered here, it lies within 0.9 to 1.1. Likewise, values of the TP from [15] greater than unity indicate twinning and here in Table 2, we see they all lie above 1.0, except Pt. We find that the twinnability ratio $\gamma_{I} / \gamma_{U}$ proposed by Jin et al. [69] and Van Swygenhoven et al. [12] exhibits more spread among the fcc metals. If we apply their suggested cut-off value of 0.8 , then $\mathrm{Al}$ and $\mathrm{Pt}$ would not twin and the rest of the metals would. It is, of course, possible to use the three aforementioned TPs to rank metals by their propensity to twin rather than make a 'twin/no twin' decision. In this case, all three TPs agree that Pt is the least likely to twin and $\mathrm{Ag}$ the most likely. However, the intermediate ranked order do not agree.

The trends predicted by Jin et al. [69] and Van Swygenhoven et al. [12] are most consistent with the present DFTPFDD predictions. Together, we predict that $\mathrm{Ag}, \mathrm{Cu}$, and $\mathrm{Rh}$ are more likely to twin than Au and Ni. Another common feature is that we find that twinnability depends on $\gamma_{I}$ but not on twin fault energies such as $\gamma_{U T}[12,10,68]$. The other TPs are based on the more commonly considered MAP model for layerby-layer construction from the same grain boundary, which introduces an additional sensitivity to twin fault energies. Experimental evaluation of twinnability in fact concluded that twin fault energies have only a limited effect on the propensity of twin nucleation [18].

As DFT-PFDD is a meso-scale model, some material details are not represented. Atomic-scale features of the grain boundaries are not modeled. Inertial effects associated with dislocation glide are missing, which may prove to be important at high rates [70]. The thermodynamic effects of temperature on energy minimization are not taken into account. At present, to simulate behavior at a given temperatures, the elastic moduli at that temperature is used. Last, we assume elastic isotropy as opposed to elastic anisotropy. In spite of these limitations, the trends reported here ought to be valid, as the governing principles of energy minimization and the balance between strain 
energy, core energies, and applied work are employed. Further, we envision that with future model development, many of these shortcomings can be overcome.

\section{Conclusions}

In this work, a density functional theory-phase field dislocation dynamics (DFT-PFDD) model is used to study stressinduced emission of defects from grain boundaries in nanoscale fcc crystals under ambient conditions. The DFT-PFDD model uniquely incorporates the full 3D $\gamma$-surface from DFT into the free energy functional on which the phase field formulation is based. The results find that the maximum grain size $D_{S F}$ below which a stacking fault and a low normalized intrinsic stacking fault energy, $\gamma_{I} / \mu b$, where $\mu$ is the shear modulus and $b$ is the value of the perfect Burgers vector are both key factors in determining whether two-layer twins will form in fcc metals. However, while grain sizes smaller than $D_{S F}$ is a necessary condition for twinning it is not sufficient. Our key finding is that deformation twinning additionally scales with $\Delta_{S F E}=$ $\left(\gamma_{U}-\gamma_{I}\right) / \mu b$, where $\gamma_{U}$ is the unstable stacking fault energy. The results are not only consistent with experimental observation but can help to explain why there are less observations of twinning in Au than $\mathrm{Cu}$ and $\mathrm{Ag}$, despite the relatively low $\gamma_{I}$ of $\mathrm{Au}$. To generalize these results to other fcc metals, a twinnability map is presented that combines the effects of the material $\gamma$-surface and nanograin sizes for several pure fcc metals. The findings may provide valuable insight for designing nanocrystalline materials for improved structural performance.

\section{Acknowledgements}

$\mathrm{AH}$ and IJB would like to acknowledge support from the Los Alamos National Laboratory Directed Research and Development (LDRD) Program through the project 20130745ECR. Los Alamos National Laboratory, an affirmative action equal opportunity employer, is operated by Los Alamos National Security, LLC, for the National Nuclear Security Administration of the US Department of Energy under Contract DE-AC5206NA25396.

\section{References}

[1] I. J. Beyerlein, X. Zhang, A. Misra, Growth twins and deformation twins in metals, Annual Review of Materials Research 44 (2014) 329-363.

[2] Y. T. Zhu, X. Z. Liao, X. L. Wu, Deformation twinning in nanocrystalline materials, Progress in Material Sciences 57 (2012) 1-62.

[3] J. P. Hirth, J. Lothe, Theory of Dislocations, 2nd Edition, Krieger Pub. Co., Florida, 1992.

[4] D. Hull, D. Bacon, Introduction to dislocations, Elsevier ButterworthHeinemann, Oxford, 2001.

[5] J. W. Christian, S. Mahajan, Deformation twinning, Progress in Material Sciences 39 (1995) 1-157.

[6] S. Mahajan, Critique of mechanisms of formation of deformation, annealing and growth twins: Face-centered cubic metals and alloys, Scripta Metallurgica 68 (2013) 95-99.

[7] S. Ogata, J. Li, S. Yip, Energy landscape of deformation twinning in bcc and fcc metals, Physical Review B 71 (224102) (2005) 1-11.
[8] X. Z. Liao, F. Zhou, E. J. Lavernia, S. G. Srinivasan, M. I. Baskes, D. W. He, Y. T. Zhu, Deformation mechanism in nanocrystalline al: Partial dislocation slip, Applied Physics Letters 83 (4) (2003) 632-634.

[9] V. Yamakov, D. Wolf, S. R. Phillpot, H. Gleiter, Deformation twinning in nanocrystalline al by molecular dynamics simulation, Acta Materialia 50 (2002) 5005-5020

[10] S. Kibey, J. B. Liu, D. D. Johnson, H. Sehitoglu, Predicting twinning stress in fcc metals: Linking twin-energy pathways to twin nucleation, Acta Materialia 55 (2007) 6843-6851.

[11] S. Kibey, J. B. Liu, D. D. Johnson, H. Sehitoglu, Energy pathways and directionality in deformation twinning, Applied Physics Letters 91 (181916) (2007) 1-3.

[12] H. Van Swygenhoven, P. M. Derlet, A. G. Frøseth, Stacking fault energies and slip in nanocrystalline metals, Nature Materials 3 (2004) 399-403.

[13] R. J. McCabe, I. J. Beyerlein, J. S. Carpenter, N. A. Mara, The critical role of grain orientation and applied stress for nanoscale twinning, Nature Communications 5 (2014) 3806.

[14] M. Chen, E. Ma, K. J. Hemker, H. Sheng, Y. Wang, X. Cheng, Deformation twinning in nanocrystalline aluminum, Science 300 (5623) (2003) 1275-1277.

[15] R. J. Asaro, S. Suresh, Mechanistic models for the activation volume and rate sensitivity in metals with nanocrystalline grains and nano-scale twins, Acta Materialia 53 (2005) 3369-3382.

[16] Y. T. Zhu, X. Z. Liao, Retaining ductility, Nature Materials 3 (2004) 351352.

[17] Y. T. Zhu, X. Z. Liao, S. G. Srinivasan, E. J. Lavernia, Nucleation and deformation twins in nanocrystalline face-centered-cubic metals by severe plastic deformation, Journal of Applied Physics 98 (034319) (2005) 1-8.

[18] X. L. Wu, Y. T. Zhu, Inverse grain-size effect on twinning in nanocrystalline ni, Physical Review Letters 101 (025503) (2008) 1-4.

[19] E. Martinez, J. Marian, A. Arsenlis, M. Victoria, J. Perlado, Atomistically informed dislocation dynamics in fcc crystals, Journal of the Mechanics and Physics of Solids 56 (2008) 869-895.

[20] J. Douin, F. Pettinari-Sturmel, A. Coujou, Dissociated dislocations in confined plasticity, Acta Materialia 55 (2007) 6453-6458.

[21] A. Hunter, R. F. Zhang, I. J. Beyerlein, T. C. Germann, M. Koslowski, Dependence of equilibrium stacking fault width in fcc metals on the $\gamma$ surface, Modelling and Simulation in Materials Science and Engineering 21 (025015) (2013) 1-19.

[22] A. Hunter, R. F. Zhang, I. J. Beyerlein, The core structure of dislocation and their relationship to the material $\gamma$-surface, Journal of Applied Physics 115 (2014) 134314.

[23] A. Hunter, I. J. Beyerlein, Stacking fault emission from grain boundaries Material dependencies and grain size effects, Materials Science and Engineering A 600 (2014) 200-210.

[24] X. L. Wu, X. Z. Liao, S. G. Srinivasan, F. Zhou, E. J. Lavernia, R. Z. Valiev, Y. T. Zhu, New deformation twinning mechanism generates zero macroscopic strain in nanocrystalline metals, Physical Review Letters 100 (095701) (2008) 1-4.

[25] M. Koslowski, A. Cuitiño, M. Ortiz, A phase-field theory of dislocations dynamics, strain hardening and hysteresis in ductile single crystals, Journal of the Mechanics and Physics of Solids 50 (12) (2002) 2597-2635.

[26] M. Koslowski, Scaling laws in plastic deformation, Philosophical Magazine 87 (8-9) (2007) 1175-1184.

[27] M. Koslowski, Effect of grain size distribution on plastic strain recovery, Physical Review B 82 (054110).

[28] A. Hunter, I. J. Beyerlein, T. C. Germann, M. Koslowski, Infuence of the stacking fault energy surface on partial dislocations in fcc metals with a three-dimensional phase field model, Physical Review B 84 (144108) (2011) 1-10.

[29] A. Hunter, I. J. Beyerlein, Predictions of an alternative pathway for grainboundary driven twinning, Applied Physics Letters 104 (233112) (2014) $1-4$.

[30] M. Koslowski, D. Wook Lee, L. Lei, Role of grain boundary energectics on the maximum strength of nano crystalline nickel, Journal of the Mechanics and Physics of Solids 59 (2011) 1427-1436.

[31] L. Lei, J. L. Marian, M. Koslowski, Phase-field modeling of defect nucleation and propagation in domains with material inhomogeneities, Modelling and Simulation in Materials Science and Engineering 21 (025009) (2013) 1-15.

[32] M. Koslowski, R. LeSar, T. Robb, Avalanches and scaling in plastic de- 
Table 2: Table of twinnability parameters calculated from relationships proposed in literature.

\begin{tabular}{|c|c|c|c|c|c|c|}
\hline Material & $\gamma_{I}[22]$ & $\gamma_{U}[22]$ & $\gamma_{U T}[69]$ & $\begin{array}{c}{\left[1.136-0.151 \frac{\gamma_{I}}{\gamma_{U}}\right] \sqrt{\frac{\gamma_{U}}{\gamma_{U T}}}[68]} \\
>1=\mathrm{twin}\end{array}$ & $\begin{array}{c}\sqrt{\left(3 \gamma_{U}-2 \gamma_{I}\right) / \gamma_{U T}}[15] \\
>1=\text { twin }\end{array}$ & $\begin{array}{l}\frac{\gamma_{I}}{\gamma_{U}}[69,12] \\
<0.8=\text { twin }\end{array}$ \\
\hline $\mathrm{Ag}$ & 17.8 & 100.4 & 100.0 & 1.11 & 1.63 & 0.18 \\
\hline $\mathrm{Al}$ & 140.2 & 177.0 & 196.0 & 0.97 & 1.13 & 0.79 \\
\hline $\mathrm{Au}$ & 27.9 & 66.5 & 79.0 & 0.98 & 1.35 & 0.42 \\
\hline $\mathrm{Cu}$ & 38.5 & 163.7 & 179.0 & 1.05 & 1.52 & 0.24 \\
\hline Ir & 324.4 & 614.9 & 818.0 & 0.92 & 1.21 & 0.53 \\
\hline $\mathrm{Ni}$ & 144.5 & 289.0 & 323.0 & 1.00 & 1.34 & 0.5 \\
\hline $\mathrm{Pd}$ & 138.1 & 197.9 & 261.0 & 0.90 & 1.10 & 0.70 \\
\hline $\mathrm{Pt}$ & 253.7 & 257.7 & 305.0 & 0.91 & 0.93 & 0.99 \\
\hline $\mathrm{Rh}$ & 184.6 & 454.1 & - & - & - & 0.41 \\
\hline
\end{tabular}

formation, Physical Review Letters 93 (12).

[33] J. P. Perdew, K. Burke, M. Ernzerhof, Generalized gradient approximation made simple, Physical Review Letters 77 (18) (1996) 3865-3868

[34] P. E. Blöchl, Projector augmented-wave method, Physical Review B 50 (24) (1994) 17953-17979.

[35] G. Kresse, D. Joubert, From ultrasoft psuedopotentials to the projector augmented-wave method, Physical Review B 59 (3) (1999) 1758-1775.

[36] H. J. Monkhorst, J. D. Pack, Special points for brillouin-zone integrations, Physical Review B 13 (12) (1976) 5188-5192.

[37] M. Methfessel, A. T. Paxton, High-precision sampling for brillouin-zone integration in metals, Physical Review B 40 (6) (1989) 3616-3621.

[38] J. Cai, F. Wang, C. Lu, Y. Y. Wang, Structure and stacking-fault energy in metals al, pd, pt, ir, and rh, Physical Review B 69 (224104) (2004) 1-4.

[39] A. Hunter, F. Saied, C. Le, M. Koslowski, Large scale 3d phase field dislocation dynamics simulations on high performance architectures, International Journal of High Performance Computing 25 (2) (2010) 223-235.

[40] L. Lei, M. Koslowski, Mesoscale modeling of dislocations in molecular crystals, Philosophical Magazine 91 (6) (2011) 865-878.

[41] Y. U. Wang, Y. M. Jin, A. M. Cuitiño, A. G. Khachaturyan, Nanoscale phase field microelasticity theory of dislocations: model and $3 \mathrm{~d}$ simulations, Acta Materialia 49 (2001) 1847-1857.

[42] D. Lee, H. Kim, A. Strachan, M. Koslowski, Effect of core energy on the mobility in a continuum dislocation model, Physical Review B 83 (2011) 104101.

[43] T. Mura, Micromechanics of defects in solids, Kluwer Academic Publishers, 1987.

[44] R. Peierls, The size of a dislocation, Proceedings of the Physical Society 52 (1) (1940) 24-37.

[45] F. Nabarro, Dislocations in a simple cubic lattice, Proceedings of the Physical Society 59 (2) (1947) 256-272.

[46] M. Ortiz, R. Phillips, Nanomechanics of defects in solids, Advances in Applied Mechanics 36 (1999) 1-79.

[47] G. Schoeck, The core structure, recombination energy and peierls energy for dislocations in al, Philosophical Magazine A 81 (5) (2001) 11611176.

[48] C. Shen, Y. Wang, Incorporation of $\gamma$-surface to phase field model of dislocation:simulating dislocation dissociation in fcc crystals, Acta Materialia 52 (2004) 683-691.

[49] M. Frigo, S. G. Johnson, The Fastest Fourier Transform in the West, Tech. Rep. MIT-LCS-TR-728, Laboratory for Computing Sciences, MIT, Cambridge, MA (1997).

[50] M. Frigo, S. G. Johnson, FFTW: An adaptive software architecture for the FFT, in: IEEE International Conference on Acoustics, Speech and Signal Processing (ICASSP), 1998, pp. 1381-1384.

[51] G. Kresse, J. Furthmüller, Efficiency of ab initio total energy calculations for metals and semiconductors using a plane-wave basis set, Computational Materials Science 6 (1) (1996) 15-50.

[52] G. Kresse, J. Furthmüller, Efficient iterative schemes for ab initio totalenergy calculations using a plane-wave basis set, Physical Review B 54 (16) (1996) 11169-11186.

[53] F. Mompiou, M. Legros, A. Bóe, M. Coulombier, J. P. Raskin, T. Pardoen, Inter- and intragranular plasticity mechanisms in ultrafine-grained al thin flims: An in situ tem study, Acta Materialia 61 (2013) 205-216.
[54] J. Godet, S. Brochard, L. Pizzagalli, P. Beauchamp, J. M. Soler, Dislocation formation from a surface step in semiconductors: An ab initio study, Physical Review B 73 (092105) (2006) 1-4.

[55] V. Navarro, O. Rodríguez de la Fuente, A. Mascaraque, J. M. Rojo, Uncommon dislocation processes at the incipient plasticity of stepped gold surfaces, Physical Review Letters 100 (105504) (2008) 1-4.

[56] T. Malis, D. J. Lloyd, K. Tangri, Dislocation generation from grain boundaries in nickel, Physica Status Solidi A 11 (1972) 275-286.

[57] A. Hunter, I. J. Beyerlein, Unprecedented grain size effect on stacking fault width, Applied Physics Letters Materials 1 (032109) (2013) 1-9.

[58] J. Rice, Dislocation nucleation from a crack tip - an analysis based on the peierls concept, Journal of the Mechanics and Physics of Solids 40 (2) (1992) 239-271.

[59] I. J. Beyerlein, N. A. Mara, D. Bhattacharyya, D. J. Alexander, C. T. Necker, Texture evolution via combined slip and deformation twinning in rolled silver-copper cast eutectic nanocomposite, International Journal of Plasticity 27 (2011) 121-146.

[60] I. J. Beyerlein, L. S. Tóth, C. N. Tóme, S. Suwas, Role of twinning on texture evolution of silver during equal channel angular extrusion, Philosophical Magazine 87 (6) (2007) 885-906.

[61] H. Suzuki, C. S. Barrett, Deformation twinning in silver-gold alloys, Acta Metallurgica 6 (1958) 156-165.

[62] Y. Yue, P. Liu, Q. Deng, E. Ma, Z. Zhang, X. Han, Quantitative evidence of crossover toward partial dislocation mediated plasticity in copper single crystal nanowires, Nano Letters 12 (2012) 4045-4049.

[63] C. X. Huang, K. Wang, S. D. Wu, Z. F. Zhang, G. Y. Li, S. X. Li, Deformation twinning in polycrystalline copper at room temperature and low strain rate, Acta Metallurgica 54 (3) (2006) 655-665.

[64] S. Oh, M. Legros, D. Kiener, P. Gruber, G. Dehm, In situ tem straining of single crystal au films on polyimide: Change of deformation mechanisms at the nanoscale, Acta Materialia 55 (2007) 5558-5571.

[65] D. H. Warner, W. A. Curtin, S. Qu, Rate dependence of crack-tip processes predicts twinning trends in f.c.c. metals, Nature Materials 6 (2007) 876-881.

[66] X. Wu, Y. T. Zhu, M. W. Chen, E. Ma, Twinning and stacking fault formation during tensile deformation of nanocrystalline ni, Scripta Materialia 54 (2006) 1685-1690.

[67] E. Tadmor, S. Hai, A peierls criterion for the onset of deformation twinning at a crack tip, Journal of the Mechanics and Physics of Solids 51 (2003) 765-793.

[68] N. Bernstein, E. Tadmor, Tight-binding calculations of stacking fault energies and twinability in fcc metals, Physical Review B 69 (094116).

[69] Z. H. Jin, S. T. Dunham, H. Gleiter, H. Hahn, P. Gumbsch, A universal scaling of planar fault energy barriers in face-centered cubic materials, Scripta Metallurgica 64 (7) (2011) 605-608.

[70] Z. Q. Wang, I. J. Beyerlein, R. LeSar, Dislocation motion in high strainrate deformation, Philosophical Magazine 87 (16) (2007) 2263-2279. 